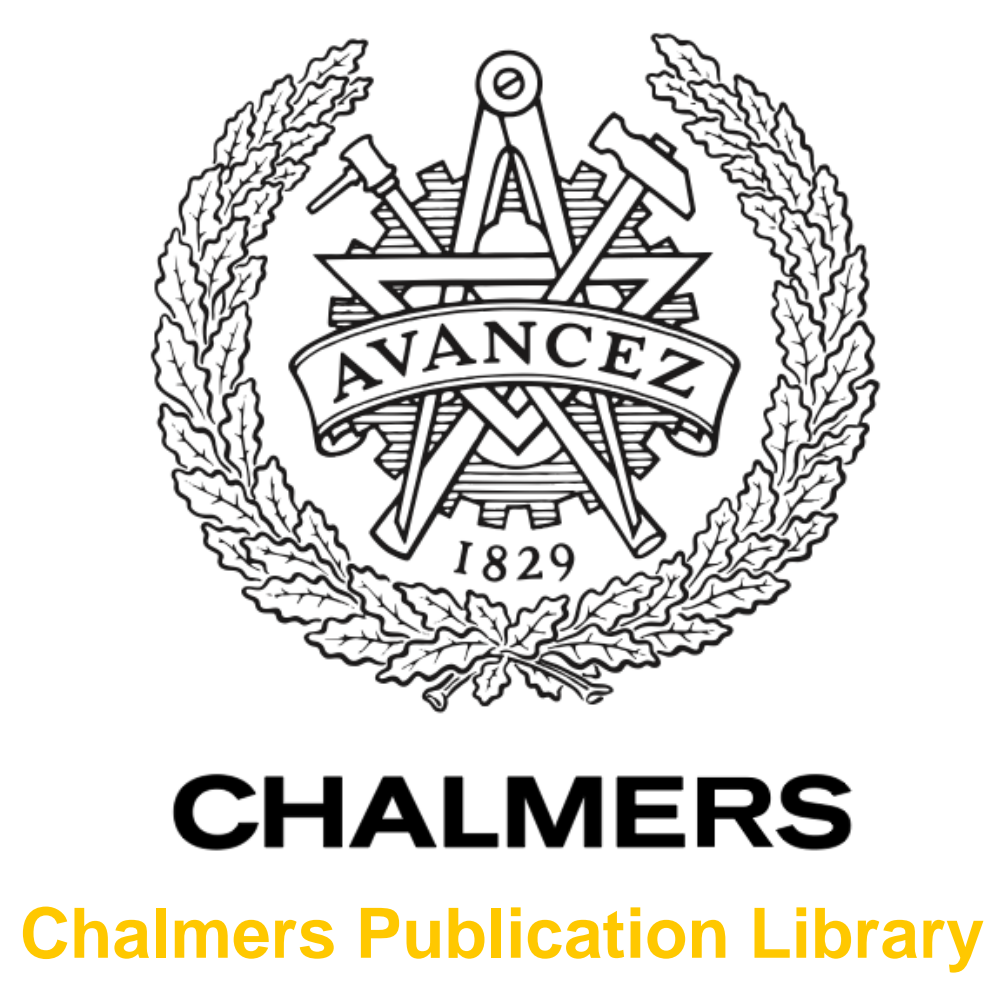

\title{
In-plane P-SV waves from a piezoelectric strip actuator: exact versus effective boundary condition solutions
}

This document has been downloaded from Chalmers Publication Library (CPL). It is the author's version of a work that was accepted for publication in:

IEEE Transactions on Ultrasonics, Ferroelectrics and Frequency Control (ISSN: 0885-3010)

Citation for the published paper:

Boström, A. ; Zhang, B. (2005) "In-plane P-SV waves from a piezoelectric strip actuator: exact versus effective boundary condition solutions". IEEE Transactions on Ultrasonics, Ferroelectrics and Frequency Control, vol. 52(9), pp. 1594-1600.

http://dx.doi.org/10.1109/TUFFC.2005.1516032

Downloaded from: http://publications.lib.chalmers.se/publication/10193

Notice: Changes introduced as a result of publishing processes such as copy-editing and formatting may not be reflected in this document. For a definitive version of this work, please refer to the published source. Please note that access to the published version might require a subscription.

Chalmers Publication Library (CPL) offers the possibility of retrieving research publications produced at Chalmers University of Technology. It covers all types of publications: articles, dissertations, licentiate theses, masters theses, conference papers, reports etc. Since 2006 it is the official tool for Chalmers official publication statistics. To ensure that Chalmers research results are disseminated as widely as possible, an Open Access Policy has been adopted.

The CPL service is administrated and maintained by Chalmers Library. 


\title{
Inplane P-SV waves from a piezoelectric strip actuator - exact versus effective boundary condition solutions
}

\author{
Anders Boström and Bixing Zhang
}

\begin{abstract}
A piezoelectric strip of finite width and thickness is placed on top of an isotropic elastic halfspace. It operates in actuator mode and a time harmonic voltage is thus applied across it. The piezoelectric material is of type $6 \mathrm{~mm}$ oriented so that a 2D inplane (P-SV) problem results. By Fourier series expansions the problem is solved exactly and this result is compared to the case when the piezoelectric strip is replaced by an effective boundary condition, which is derived by series expansions in the thickness coordinate in the piezoelectric strip. At low frequencies the results agree very well and this corresponds to the situation often met in practice. In general, the effective boundary condition should be much easier to apply, for example when a FEM program is used.
\end{abstract}

\section{INTRODUCTION}

A CTUATION and sensing in structures is often performed with piezoelectric materials, and in this connection it is common to talk about "smart" structures in, e.g., structural control and noise control applications. The piezoelectric material is usually in the shape of a thin layer that is surface bonded to or embedded in the structure. Much interest has therefore been given to the modeling of such piezoelectric layers. Gopinathan et al. [1] give a recent review with particular emphasis on various approximate plate theories and make comparisons with exact 3D solutions for a beam fully covered with a piezoelectric layer. A few other 3D investigations of piezoelectric vibration problems in more or less simplified situations have been performed. Batra et al. [2] consider a laminated rectangular plate with piezoelectric coatings which are treated by a 2D theory due to Tiersten [3]. Gao et al. [4] consider a very similar problem but treat the piezoelectric coatings by $3 \mathrm{D}$ theory. Both these investigations, and also Gopinahtan et al. [1], use

A. Boström is with Chalmers University of Technology, Göteborg, Sweden. mixed boundary conditions (involving both displacements and stresses) to model a simply supported plate. This is in fact necessary for the success of the Fourier series method adopted. Clamped or completely free edges can not be treated by the method employed.

Also various approximate plate and shell theories have been developed for layered structures including piezoelectrics, several such theories are cited by Gopinahtan et al. [1]. In a recent review article, Wang and Yang [5] discuss higher-order piezoelectric plate theories and their applications. The finite element method is developed for such problems by, e.g., Tzou [6] and Kim et al. [7].

Another approach to model a thin piezoelectric layer is to exchange it with an effective (or approximate) boundary condition. In this way no equations of motion for the piezoelectric layer have to be considered at all. This can greatly simplify the analysis and could be particularly useful in FEM applications, where commercial codes often do not include the option of a piezoelectric material. Johansson and Niklasson [8] develop such effective boundary conditions and make comparisons with exact calculations for 1D problems and a 2D plate problem. Only cases with an infinite structure are considered, but it is of course important to see how these effective boundary conditions perform for finite piezoelectric patches. The purpose of the present paper is to do exactly this. Thus the 2D problem with a finite piezoelectric strip on a halfspace is considered for coupled P-SV motion. It is noted that the corresponding antiplane $(\mathrm{SH})$ case has already been treated by Zhang et. al [9].

The plan of the paper is as follows. In Section 2 the problem is formulated. A piezoelectric strip of finite width and thickness is attached to an isotropic elastic half-space. The piezoelectric material is of class $6 \mathrm{~mm}$ poled in the thickness direction, so that a 
2D inplane (P-SV) problem including piezoelectric effects is possible. An actuator case is studied and a time harmonic voltage is thus applied across the piezoelectric strip. In Section 3 the problem is solved exactly by expanding the fields inside the piezoelectric strip in Fourier series and the field in the half-space in Fourier integrals. Applying all the boundary conditions, it is straightforward to obtain a system of equations for the expansion coefficients in the strip. In Section 4 the boundary value problem with the effective boundary condition is solved. In general the effective boundary condition involves both the stress and displacement and the boundary value problem is thus of mixed type. It is solved in a way very similar to the exact problem. Section 5 contains numerical examples with comparisons between the exact solution and various orders of the effective boundary condition. As expected the effective boundary condition only yields accurate results at low frequencies.

\section{PRoblem FORMUlation}

Consider the 2D in-plane wave propagation problem depicted in Fig. 1. In the $x z$ plane an isotropic elastic medium occupies the half-space $z<0$ and on top of the half-space a piezoelectric strip occupies the region $|x| \leq a$ and $0 \leq z \leq h$. Only time harmonic conditions are considered and the time factor $\exp (-\mathrm{i} \omega t)$, where $\omega$ is the angular frequency and $t$ is time, is suppressed throughout. In the half-space the displacement components are $u^{e}$ and $w^{e}$ in the $x$ and $z$ direction, respectively, and in the piezoelectric strip the corresponding components are $u$ and $w$.

The isotropic half-space has density $\rho^{e}$ and Lamé constants $\lambda$ and $\mu$. The stress components are then

$$
\begin{gathered}
\sigma_{x x}^{e}=(\lambda+2 \mu) \partial_{x} u^{e}+\lambda \partial_{z} w^{e} \\
\sigma_{z z}^{e}=\lambda \partial_{x} u^{e}+(\lambda+2 \mu) \partial_{z} w^{e}, \\
\sigma_{x z}^{e}=\mu\left(\partial_{z} u^{e}+\partial_{x} w^{e}\right)
\end{gathered}
$$

where partial derivatives with respect to $x$ and $z$ are denoted by $\partial_{x}$ and $\partial_{z}$, respectively. The equations of motion are

$$
\begin{aligned}
(\lambda+2 \mu) \partial_{x}^{2} u^{e} & +(\lambda+\mu) \partial_{x} \partial_{z} w^{e} \\
& +\mu \partial_{z}^{2} u^{e}=-\rho^{e} \omega^{2} u^{e}
\end{aligned}
$$

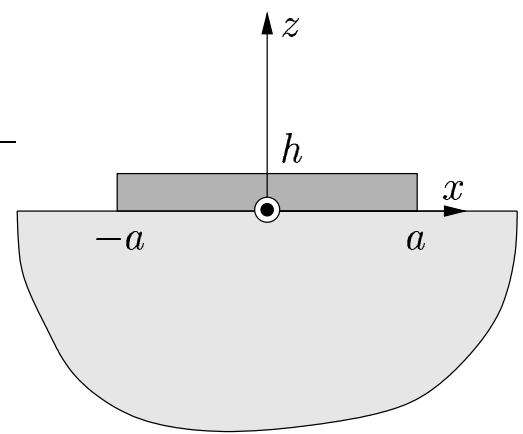

Fig. 1. The 2D geometry with a piezoelectric strip actuator on an isotropic half-space.

$$
\begin{aligned}
(\lambda+2 \mu) \partial_{z}^{2} w^{e} & +(\lambda+\mu) \partial_{x} \partial_{z} u^{e} \\
& +\mu \partial_{x}^{2} w^{e}=-\rho^{e} \omega^{2} w^{e} .
\end{aligned}
$$

The piezoelectric strip is assumed to be of class $6 \mathrm{~mm}$ and poled in the $z$ direction. It has density $\rho$ and the only relevant stiffness constants are $c_{11}, c_{33}$, $c_{13}$, and $c_{44}$, in abbreviated notation, cf. Auld [10]. The relevant piezoelectric coupling constants are $e_{15}, e_{31}$, and $e_{33}$, and the dielectric constants are $\epsilon_{11}$ and $\epsilon_{33}$. The quasistatic approximation is made and the electric potential is $\phi$. The stress components are

$$
\begin{aligned}
\sigma_{x x} & =c_{11} \partial_{x} u+c_{13} \partial_{z} w+e_{31} \partial_{z} \phi, \\
\sigma_{z z} & =c_{13} \partial_{x} u+c_{33} \partial_{z} w+e_{33} \partial_{z} \phi, \\
\sigma_{x z} & =c_{44}\left(\partial_{z} u+\partial_{x} w\right)+e_{15} \partial_{x} \phi,
\end{aligned}
$$

and the two electric displacement components are

$$
\begin{aligned}
& D_{x}=e_{31} \partial_{z} u+e_{15} \partial_{x} w-\epsilon_{11} \partial_{x} \phi \\
& D_{z}=e_{15} \partial_{x} u+e_{33} \partial_{z} w-\epsilon_{33} \partial_{z} \phi .
\end{aligned}
$$

The three equations of motion for the strip become

$$
\begin{gathered}
c_{11} \partial_{x}^{2} u+c_{44} \partial_{z}^{2} u+\left(c_{13}+c_{44}\right) \partial_{x} \partial_{z} w \\
+\left(e_{15}+e_{31}\right) \partial_{x} \partial_{z} \phi=-\rho \omega^{2} u, \\
c_{44} \partial_{x}^{2} w+c_{33} \partial_{z}^{2} w+\left(c_{13}+c_{44}\right) \partial_{x} \partial_{z} u \\
+e_{15} \partial_{x}^{2} \phi+e_{33} \partial_{z}^{2} \phi=-\rho \omega^{2} w \\
e_{15} \partial_{x}^{2} w+e_{33} \partial_{z}^{2} w+\left(e_{15}+e_{31}\right) \partial_{x} \partial_{z} u \\
-\epsilon_{11} \partial_{x}^{2} \phi-\epsilon_{33} \partial_{z}^{2} \phi=0 .
\end{gathered}
$$

Due to the quasistatic approximation this system is not hyperbolic, of course.

The piezoelectric strip is electroded on the top and bottom boundaries. The electrodes are assumed 
to be perfectly conducting but to have negligible mechanical influence. The situation when the piezoelectric strip acts as an actuator is of interest so a (time harmonic) driving voltage $V_{0}$ across the strip leads to the boundary conditions

$$
\begin{array}{ll}
\phi=0, & z=0, \quad|x| \leq a, \\
\phi=V_{0}, \quad z=h, & |x| \leq a .
\end{array}
$$

Only the potential difference is of importance, of course. On the sides of the piezoelectric strip there are no electrodes and an approximate boundary condition for this is (see Tiersten [11])

$$
D_{x}=0, \quad x= \pm a, \quad 0 \leq z \leq h,
$$

where $D_{x}$ is given in Eq. (9).

The mechanical boundary conditions on the sides of the piezoelectric strip are chosen as

$$
\begin{array}{rll}
u=0, & x= \pm a, & 0 \leq z \leq h, \\
\sigma_{x z}=0, & x= \pm a, & 0 \leq z \leq h .
\end{array}
$$

This choice may seem a little odd and not very realistic. It is also used by Gopinathan et al. [1], Batra et al. [2], and Gao et el. [4], but then to model the boundary conditions for a plate. However, the piezoelectric strip is thin and then it can be argued that the exact boundary conditions on the sides are not very important. This will be further commented on when the exact and effective boundary conditions are compared. The great advantage with the boundary conditions (17) and (18) is that they together with the boundary condition (16) lead to the following simpler boundary conditions

$$
\begin{array}{rll}
u=0, & x= \pm a, & 0 \leq z \leq h, \\
\partial_{x} w=0, & x= \pm a, & 0 \leq z \leq h, \\
\partial_{x} \phi=0, & x= \pm a, & 0 \leq z \leq h .
\end{array}
$$

This will make it possible to expand the fields inside the piezoelectric strip in simple trigonometric series in $x$.

On top of the piezoelectric strip the traction should vanish

$$
\begin{aligned}
& \sigma_{z z}=0, \quad z=h, \quad|x| \leq a, \\
& \sigma_{x z}=0, \quad z=h, \quad|x| \leq a .
\end{aligned}
$$

Likewise, on top of the elastic half-space outside the strip

$$
\begin{aligned}
& \sigma_{z z}=0, \quad z=0, \quad|x|>a, \\
& \sigma_{x z}=0, \quad z=0, \quad|x|>a \text {. }
\end{aligned}
$$

Between the piezoelectric strip and the elastic half-space the displacement and traction should be continuous

$$
\begin{array}{ll}
u=u^{e}, & z=0, \quad|x| \leq a, \\
w=w^{e}, & z=0, \quad|x| \leq a, \\
\sigma_{z z}=\sigma_{z z}^{e}, & z=0, \quad|x| \leq a, \\
\sigma_{x z}=\sigma_{x z}^{e}, & z=0, \quad|x| \leq a .
\end{array}
$$

To completely specify the problem the radiation condition that the waves are down-going in the halfspace must be imposed. There will also be outgoing Rayleigh waves along the surface of the half-space.

\section{EXACT SOLUTION}

In the isotropic elastic half-space $z<0$ the waves can be represented by Fourier integrals as

$$
\begin{gathered}
u^{e}=\int_{-\infty}^{\infty}\left(q A \mathrm{e}^{-\mathrm{i} p_{p} z}+p_{s} B \mathrm{e}^{-\mathrm{i} p_{s} z}\right) \mathrm{e}^{\mathrm{i} q x} \mathrm{~d} q, \\
w^{e}=\int_{-\infty}^{\infty}\left(-p_{p} A \mathrm{e}^{-\mathrm{i} p_{p} z}+q B \mathrm{e}^{-\mathrm{i} p_{s} z}\right) \mathrm{e}^{\mathrm{i} q x} \mathrm{~d} q .
\end{gathered}
$$

Here $A=A(q)$ and $B=B(q)$ are the unknown amplitudes of the pressure and shear waves, respectively. The wave numbers in the $z$ direction are $p_{p}=\sqrt{k_{p}^{2}-q^{2}}$ and $p_{s}=\sqrt{k_{s}^{2}-q^{2}}$ with the square roots defined so that $\operatorname{Im} p_{p} \geq 0$ and $\operatorname{Im} p_{s} \geq 0$. The wave numbers are $k_{p}=\omega \sqrt{\rho^{e} /(\lambda+2 \mu)}$ and $k_{s}=$ $\omega \sqrt{\rho^{e} / \mu}$. Note that the radiation condition has been used when writing the representation given in Eqs. (30) and (31). From Eqs. (1)-(3) the corresponding stresses in the half-space are easily determined.

In the piezoelectric strip the situation is more complicated. Due to the boundary conditions Eqs. (19)-(21) and the fact that the solution must be symmetric in $x$ (this symmetry for the displacement vector means that $u$ must be an odd function and $w$ an even function of $x$ ), it is possible to expand the fields in trigonometric series in the following way

$$
\begin{aligned}
u & =\sum_{m=1}^{\infty} u_{m}(z) \sin q_{m} x \\
w & =\sum_{m=0}^{\infty} w_{m}(z) \cos q_{m} x \\
\phi & =\sum_{m=0}^{\infty} \phi_{m}(z) \cos q_{m} x
\end{aligned}
$$


where $q_{m}=m \pi / a$. The three equations of motion (11)-(13) are then reduced to a set of ordinary differential equations in $z$ for each $m$. For $m=0$ the solution is

$$
\begin{gathered}
w_{0}=C_{01} \cos k_{0} z+D_{01} \sin k_{0} z \\
\phi_{0}=\frac{e_{33}}{\epsilon_{33}}\left(C_{01} \cos k_{0} z+D_{01} \sin k_{0} z\right)+C_{02}+D_{02} z,
\end{gathered}
$$

where $C_{01}, D_{01}, C_{02}, D_{02}$ are constants to be determined and the stiffened wave number is $k_{0}=$ $\omega \sqrt{\rho / \bar{c}_{33}}$ with $\bar{c}_{33}=c_{33}\left(1+e_{33}^{2} /\left(c_{33} \epsilon_{33}\right)\right)$. For $m=1,2, \ldots$ the situation is more complicated as the horizontal displacement $u_{m}$ also enters. The solution can be written as

$$
\begin{aligned}
& u_{m}=\sum_{n=1}^{3} \alpha_{m n}\left(C_{m n} \sinh p_{m n} z+D_{m n} \cosh p_{m n} z\right), \\
& w_{m}=\sum_{n=1}^{3} \beta_{m n}\left(C_{m n} \cosh p_{m n} z+D_{m n} \sinh p_{m n} z\right), \\
& \phi_{m}=\sum_{n=1}^{3} \gamma_{m n}\left(C_{m n} \cosh p_{m n} z+D_{m n} \sinh p_{m n} z\right),
\end{aligned}
$$

where $p_{m n}, \alpha_{m n}, \beta_{m n}, \gamma_{m n}$ are determined from

$$
\begin{gathered}
\left(-c_{11} q_{m}^{2}+c_{44} p_{m}^{2}+\rho \omega^{2}\right) \alpha_{m}-\left(c_{13}+c_{44}\right) q_{m} p_{m} \beta_{m} \\
-\left(e_{15}+e_{31}\right) q_{m} p_{m} \gamma_{m}=0 \\
\left(c_{13}+c_{44}\right) q_{m} p_{m} \alpha_{m}+\left(c_{33} p_{m}^{2}-c_{44} q_{m}^{2}+\rho \omega^{2}\right) \beta_{m} \\
+\left(e_{33} p_{m}^{2}-e_{15} q_{m}^{2}\right) \gamma_{m}=0 \\
\left(e_{15}+e_{31}\right) q_{m} p_{m} \alpha_{m}+\left(e_{33} p_{m}^{2}-e_{15} q_{m}^{2}\right) \beta_{m} \\
+\left(\epsilon_{11} q_{m}^{2}-\epsilon_{33} p_{m}^{2}\right) \gamma_{m}=0 .
\end{gathered}
$$

For this homogeneous system to have a solution the determinant must vanish and this yields a third order equation in $p_{m}$ which gives the three $p_{m}=p_{m n}$, $n=1,2,3$ (the sign of $p_{m n}$ does not matter, choose $\left.\operatorname{Re} p_{m n} \geq 0\right)$. The corresponding $\alpha_{m n}, \beta_{m n}, \gamma_{m n}$ can then be solved for and normalized in some convenient way. The coefficients $C_{m n}$ and $D_{m n}$ are determined below by the boundary and interface conditions.
The two electric boundary conditions (14) and (15) give

$$
\begin{gathered}
\frac{e_{33}}{\epsilon_{33}} C_{01}+C_{02}=0, \\
\sum_{n=1}^{3} C_{m n} \gamma_{m n}=0,
\end{gathered}
$$

$\frac{e_{33}}{\epsilon_{33}}\left(C_{01} \cos k_{0} h+D_{01} \sin k_{0} h\right)+C_{02}+D_{02} h=V_{0}$

$$
\sum_{n=1}^{3} \gamma_{m n}\left(C_{m n} \cosh p_{m n} h+D_{m n} \sinh p_{m n} h\right),
$$

where $m=1,2, \ldots$ The two boundary conditions (22) and (23) that the traction vanishes on top of the piezoelectric strip give

$$
\bar{c}_{33} k_{0}\left(-C_{01} \sin k_{0} h+D_{01} \cos k_{0} h\right)+e_{33} D_{02}=0,
$$

$$
\sum_{n=1}^{3} \xi_{m n}\left(C_{m n} \cosh p_{m n} h+D_{m n} \sinh p_{m n} h\right)=0,
$$

$$
\sum_{n=1}^{3} \eta_{m n}\left(C_{m n} \sinh p_{m n} h+D_{m n} \cosh p_{m n} h\right)=0
$$

where $m=1,2, \ldots$ Here

$$
\begin{gathered}
\xi_{m n}=c_{13} \alpha_{m n} q_{m}+c_{33} \beta_{m n} p_{m n}+e_{33} \gamma_{m n} p_{m n}, \\
\eta_{m n}=c_{44} \alpha_{m n} p_{m n}-c_{44} \beta_{m n} q_{m}-e_{15} \gamma_{m n} q_{m} .
\end{gathered}
$$

Note that the boundary condition on $\sigma_{x z}$ is trivially satisfied for $m=0$.

The seven Eqs. (43)-(49) are all discrete equations valid for $m=0,1,2, \ldots$. The remaining conditions, on the other hand, give functional equations valid for a certain range in $x$. The continuity of displacements, Eqs. (26) and (27), yield

$$
\begin{aligned}
\int_{-\infty}^{\infty}\left(q A+p_{s} B\right) \mathrm{e}^{\mathrm{i} q x} \mathrm{~d} q \\
=\sum_{m=1}^{\infty} \sum_{n=1}^{3} \alpha_{m n} D_{m n} \sin q_{m} x, \quad|x| \leq a
\end{aligned}
$$




$$
\begin{aligned}
\int_{-\infty}^{\infty} & \left(-p_{p} A+q B\right) \mathrm{e}^{\mathrm{i} q x} \mathrm{~d} q \\
& =C_{01}+\sum_{m=1}^{\infty} \sum_{n=1}^{3} \beta_{m n} C_{m n} \cos q_{m} x, \quad|x| \leq a .
\end{aligned}
$$

Finally, the traction boundary conditions Eqs. (24), (25), (28), and (29) can be combined to give

$$
\begin{aligned}
& \mathrm{i} \mu \int_{-\infty}^{\infty}\left(\left(k_{s}^{2}-2 q^{2}\right) A-2 q p_{s} B\right) \mathrm{e}^{\mathrm{i} q x} \mathrm{~d} q \\
& = \begin{cases}0, & |x|>a, \\
\sum_{m=0}^{\infty} \sum_{n=1}^{3} \xi_{m n} D_{m n} \cos q_{m} x, & |x| \leq a,\end{cases} \\
& -\mathrm{i} \mu \int_{-\infty}^{\infty}\left(2 q p_{p} A+\left(k_{s}^{2}-2 q^{2}\right) B\right) \mathrm{e}^{\mathrm{i} q x} \mathrm{~d} q \\
& = \begin{cases}0, & |x|>a, \\
\sum_{m=1}^{\infty} \sum_{n=1}^{3} \eta_{m n} C_{m n} \sin q_{m} x, & |x| \leq a,\end{cases}
\end{aligned}
$$

where $m=0$ is incorporated by defining

$$
\xi_{01}=\bar{c}_{33} k_{0}, \quad \xi_{02}=e_{33}, \quad \xi_{03}=0 .
$$

Inverting the Fourier transforms in Eqs. (54) and (55) and solving for $A$ and $B$ yields

$$
\begin{aligned}
A= & \frac{\mathrm{i}}{\mu R}\left[2 q p_{s} \sum_{m=1}^{\infty} \sum_{n=1}^{3} \eta_{m n} C_{m n} g_{m}(q)\right. \\
& \left.-\left(k_{s}^{2}-2 q^{2}\right) \sum_{m=0}^{\infty} \sum_{n=1}^{3} \xi_{m n} D_{m n} f_{m}(q)\right], \\
B= & \frac{\mathrm{i}}{\mu R}\left[\left(k_{s}^{2}-2 q^{2}\right) \sum_{m=1}^{\infty} \sum_{n=1}^{3} \eta_{m n} C_{m n} g_{m}(q)\right. \\
& \left.+2 q p_{r} \sum_{m=0}^{\infty} \sum_{n=1}^{3} \xi_{m n} D_{m n} f_{m}(q)\right],
\end{aligned}
$$

where

$$
\begin{aligned}
f_{m}(q) & =\frac{1}{2 \pi} \int_{-a}^{a} \cos q_{m} x \mathrm{e}^{-\mathrm{i} q x} \mathrm{~d} x \\
& =\frac{(-1)^{m} q \sin q a}{\pi\left(q^{2}-q_{m}^{2}\right)} \\
g_{m}(q) & =\frac{1}{2 \pi} \int_{-a}^{a} \sin q_{m} x \mathrm{e}^{-\mathrm{i} q x} \mathrm{~d} x \\
& =\frac{\mathrm{i}(-1)^{m} q_{m} \sin q a}{\pi\left(q^{2}-q_{m}^{2}\right)},
\end{aligned}
$$

and the Rayleigh function $R=4 q^{2} p_{p} p_{s}+\left(2 q^{2}-k_{s}^{2}\right)^{2}$. Extraction of the Fourier coefficients from Eqs. (52) and (53) yields

$$
\begin{gathered}
\sum_{n=1}^{3} \alpha_{m n} D_{m n}=-\frac{2 \pi}{a} \int_{-\infty}^{\infty}\left(q A+p_{s} B\right) g_{m}(q) \mathrm{d} q \\
C_{01}=\frac{\pi}{a} \int_{-\infty}^{\infty}\left(-p_{p} A+q B\right) f_{0}(q) \mathrm{d} q \\
\sum_{n=1}^{3} \beta_{m n} C_{m n}=\frac{2 \pi}{a} \int_{-\infty}^{\infty}\left(-p_{p} A+q B\right) f_{m}(q) \mathrm{d} q
\end{gathered}
$$

where $m=1,2, \ldots$

Inserting $A$ and $B$ from Eqs. (54) and (55) into Eqs. (61)-(63), the remaining equations for $C_{m n}$ and $D_{m n}$, Eqs. (43)-(49) and (61)-(63), form a system of linear equations that it is straightforward to solve. However, the coefficients in Eqs. (61)-(63) contain infinite integrals over $q$ that need some care in the computation.

\section{EFFECTIVE BOUNDARY CONDITIONS}

If the thickness of the piezoelectric strip $h$ is small compared to the wavelenghts, approximating the strip in a simple fashion should be possible. In this section, an effective (approximate) boundary condition taken from Johansson and Nicklasson [8] is stated and the corresponding boundary value problem is solved in a manner that is quite similar to the method used in the previous section.

With a general time dependence and a driving voltage that may depend on $x$ the form of the effective boundary conditions including linear terms in $h$ is

$$
\begin{aligned}
\sigma_{x z}^{e}= & -\left(e_{31}-e_{33} \frac{c_{13}}{c_{33}}\right) \frac{\partial}{\partial x} V_{0} \\
& -h\left[\rho \frac{\partial^{2}}{\partial t^{2}}-\left(c_{11}-\frac{c_{13}^{2}}{c_{33}}\right) \frac{\partial^{2}}{\partial x^{2}}\right] u^{e} \\
\sigma_{z z}^{e}=- & \frac{h}{2}\left[\left(e_{31}-e_{33} \frac{c_{13}}{c_{33}}\right) \frac{\partial^{2}}{\partial x^{2}}+\frac{\rho e_{33}}{c_{33}} \frac{\partial^{2}}{\partial t^{2}}\right] V_{0} \\
& -h \rho \frac{\partial^{2} w^{e}}{\partial t^{2}}
\end{aligned}
$$


In the present case with harmonic time dependence and a constant $V_{0}$ this is very much simplified

$$
\begin{array}{r}
\sigma_{x z}^{e}=h\left[\rho \omega^{2}+\left(c_{11}-\frac{c_{13}^{2}}{c_{33}}\right) \frac{\partial^{2}}{\partial x^{2}}\right] u^{e}, \\
\sigma_{z z}^{e}=\frac{h \rho \omega^{2} e_{33}}{2 c_{33}} V_{0}+h \rho \omega^{2} w^{e} .
\end{array}
$$

The lowest order approximation is to only keep the $V_{0}$ term and this is henceforth called the first order approximation. From this it is clear that the whole solution contains a factor $h$ so incorporating the $u^{e}$ and $w^{e}$ terms gives a second order solution. Also a third order solution is considered in the following; it is quite complicated so the explicit formulas are not shown, see Johansson and Niklasson [8] for a prescription for its calculation. In summary the boundary conditions up to third order can be written

$$
\begin{gathered}
\Delta_{x z}\left(\partial_{x}^{2}\right) \sigma_{x z}^{e}=\Delta_{u}\left(\partial_{x}^{2}\right) u^{e}, \\
\Delta_{z z}\left(\partial_{x}^{2}\right) \sigma_{z z}^{e}=\Delta_{V} V_{0}+\Delta_{w}\left(\partial_{x}^{2}\right) w^{e},
\end{gathered}
$$

where the various $\Delta: \mathrm{s}$ are given by a comparison with the specific orders above. As $V_{0}$ is independent of $x, \Delta_{V}$ is a constant as indicated whereas the other $\Delta:$ s are operators in $\partial_{x}^{2}$. For a boundary condition of general order there must in addition be a term in $w^{e}$ in Eq. (68) and a term in $u^{e}$ in Eq. (69).

The solution procedure is very similar to the one used in the previous section. The general solution in the elastic half-space is given by Eqs. (30) and (31). At $z=0,|x| \leq a$, the solution is expanded in trigonometric series in $x$ as

$$
\begin{array}{ll}
u^{e}(x, 0)=\sum_{m=1}^{\infty} C_{m} \sin q_{m} x, & |x| \leq a, \\
w^{e}(x, 0)=\sum_{m=0}^{\infty} D_{m} \cos q_{m} x, & |x| \leq a,
\end{array}
$$

where $q_{m}=m \pi / a$ as before. Equating with Eqs. (30) and (31), the expansion coefficients $C_{m}$ and $D_{m}$ are obtained as

$$
\begin{gathered}
C_{m}=\frac{2 \pi}{a} \int_{-\infty}^{\infty}\left(q A+p_{s} B\right) g_{m}(q) \mathrm{d} q, \\
D_{m}=\frac{\pi \epsilon_{m}}{a} \int_{-\infty}^{\infty}\left(-p_{p} A+q B\right) f_{m}(q) \mathrm{d} q .
\end{gathered}
$$

Here $\epsilon_{0}=1, \epsilon_{m}=2, m=1,2, \ldots$, is the Neumann factor and $f_{m}$ and $g_{m}$ are given in Eqs. (59) and
(60). The boundary conditions at $z=0$ may be rewritten as

$$
\begin{gathered}
\Delta_{x z}\left(\partial_{x}^{2}\right) \sigma_{x z}^{e}= \begin{cases}\Delta_{u}\left(\partial_{x}^{2}\right) u^{e}, & |x| \leq a \\
0, & |x|>a,\end{cases} \\
\Delta_{z z}\left(\partial_{x}^{2}\right) \sigma_{z z}^{e}= \begin{cases}\Delta_{V} V_{0}+\Delta_{w}\left(\partial_{x}^{2}\right) w^{e}, & |x| \leq a, \\
0, & |x|>a .\end{cases}
\end{gathered}
$$

Inserting the stresses calculated from Eq. (30) and (31) and the surface displacement expansions (70) and (71) into these equations, inverting the Fourier transforms, and solving gives

$$
\begin{gathered}
A(q)=\frac{\mathrm{i}}{\mu R}\left[\frac{2 q p_{s}}{\Delta_{x z}\left(-q^{2}\right)} \sum_{m=1}^{\infty} \Delta_{u}\left(-q_{m}^{2}\right) C_{m} g_{m}(q)\right. \\
\left.-\frac{\left(k_{s}^{2}-2 q^{2}\right)}{\Delta_{z z}\left(-q^{2}\right)}\left(\Delta_{V} V_{0}+\sum_{m=0}^{\infty} \Delta_{w}\left(-q_{m}^{2}\right) D_{m} f_{m}(q)\right)\right] \\
B(q)=\frac{\mathrm{i}}{\mu R}\left[\frac{k_{s}^{2}-2 q^{2}}{\Delta_{x z}\left(-q^{2}\right)} \sum_{m=1}^{\infty} \Delta_{u}\left(-q_{m}^{2}\right) C_{m} g_{m}(q)\right. \\
\left.+\frac{\left(q p_{p}\right.}{\Delta_{z z}\left(-q^{2}\right)}\left(\Delta_{V} V_{0}+\sum_{m=0}^{\infty} \Delta_{w}\left(-q_{m}^{2}\right) D_{m} f_{m}(q)\right)\right]
\end{gathered}
$$

where it is assumed that $\Delta_{x z}\left(-q^{2}\right) \neq 0$ and $\Delta_{z z}\left(-q^{2}\right) \neq 0$. For the first and second order boundary conditions this is certainly no restriction as $\Delta_{x z} \equiv 1$ and $\Delta_{z z} \equiv 1$, but it seems to be no restriction in general for reasonably low frequencies.

If $A$ and $B$ from Eqs. (76) and (77) are inserted into Eqs. (72) and (73) a linear system of equations is obtained for $C_{m}$ and $D_{m}$ that it is straightforward to solve. As for the exact solution the coefficients in the system of equations contain infinite integrals over $q$ that need some care in the computation.

Once the expansion coefficients $C_{m}$ and $D_{m}$ have been determined, the displacement field in the elastic half-space is obtained from Eqs. (30) and (31) (using also Eqs. (76) and (77)). Note that if the displacement directly beneath the strip $(z=0$, $|x| \leq a)$ is wanted, the series expansions (70) and (71) may be used instead. 


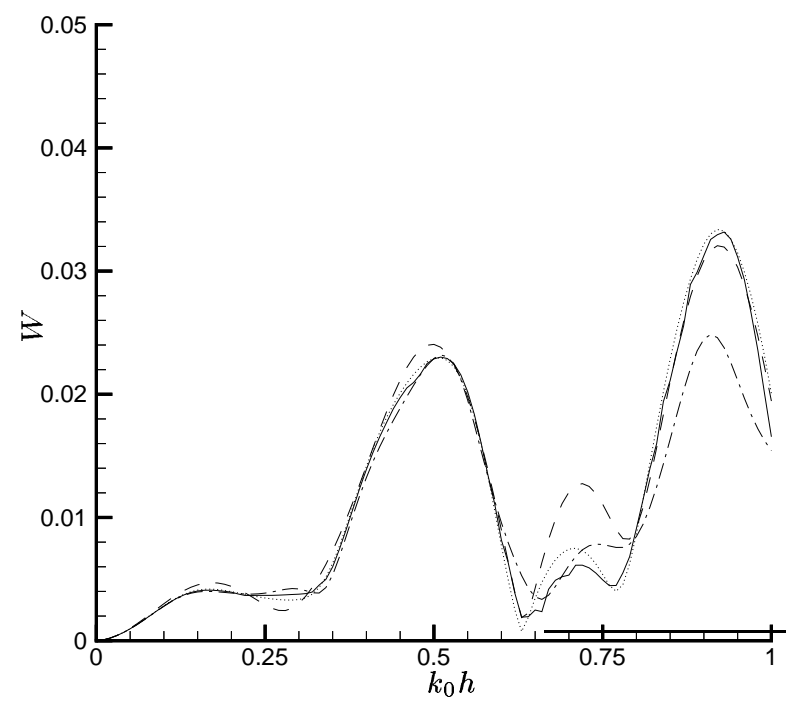

Fig. 2. The dimensionless displacement $W$ at $x=0, z=0$ as a function of frequency for $h / a=0.05$ and four cases: exact (fulldrawn), first order (dashed), second order (dash-dotted), and third order (dotted)

\section{NUMERICAL EXAMPLES}

In this section, some numerical examples are given in order to illustrate the usefulness and limitations of the effective boundary conditions. In all the examples the elastic half-space is made of steel with the material properties $\mu=80.8 \mathrm{GPa}, \lambda=121 \mathrm{GPa}$, and $\rho^{e}=7870 \mathrm{~kg} / \mathrm{m}^{3}$. The piezoelectric strip is made of PZT-2 (class $6 \mathrm{~mm}$ with the $x y$-plane as the plane of isotropy) with the material properties $c_{11}=135 \mathrm{GPa}, c_{13}=68.1 \mathrm{GPa}, c_{33}=113 \mathrm{GPa}$, $c_{44}=22.2 \mathrm{GPa}, e_{15}=9.8 \mathrm{C} / \mathrm{m}^{2}, e_{31}=-1.9 \mathrm{C} / \mathrm{m}^{2}$, $e_{33}=9.0 \mathrm{C} / \mathrm{m}^{2}, \epsilon_{11}=478 \cdot 10^{-11} \mathrm{C} / \mathrm{Vm}, \epsilon_{33}=$ $230 \cdot 10^{-11} \mathrm{C} / \mathrm{Vm}$, and $\rho=7600 \mathrm{~kg} / \mathrm{m}^{3}$ (see Auld [10]). In all the examples, the dimensionless displacement in the $z$ direction $W=\sqrt{c_{44} / \epsilon_{11}} w^{e} / V_{0}$ on the surface of the half-space is shown for the exact solution as well as the approximate solutions obtained from the effective boundary conditions. Both the frequency $k_{0} h$ of the input voltage and the thickness to width ratio $h / a$ of the strip are varied. It is noted that the dimensionless frequency is chosen as the thickness of the piezoelectric strip relative its (stiffened) wavelength as it is to be expected that it is this ratio that is crucial in determining the accuracy of the effective boundary condition.

In Figs. 2-4 the dimensionless displacement $W$ is shown at $x=0, z=0$ as a function of the

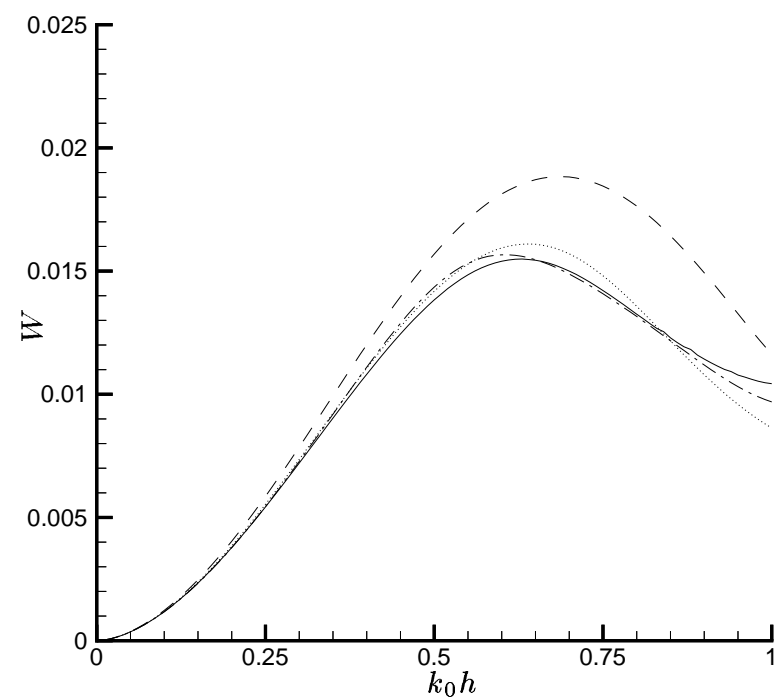

Fig. 3. The dimensionless displacement $W$ at $x=0, z=0$ as a function of frequency for $h / a=0.2$ and four cases: exact (fulldrawn), first order (dashed), second order (dash-dotted), and third order (dotted).

frequency $k_{0} h$. Between the three plots, the value of $h / a$ is varied. It is seen that the first order approximation is in good agreement with the exact solution when $k h_{0}<0.1-0.4$, depending on the value of $h / a$. The second and third order solutions are valid for somewhat higher frequencies, also somewhat depending on $h / a$. In Fig. 4 the third order solution is valid up to $k_{0} h=1$, but then it, somewhat unexpectedly, quickly deviates and even goes to zero at $k_{0} h=1.4$. However, it is doubtful if the higher order effective boundary conditions are worth the extra effort, because the boundary conditions are much more complicated, involving also tangential derivatives.

In the exact solution the boundary conditions on the sides $x= \pm a$ are of the mixed type, somewhat unrealistic in practice. In the solution with the effective boundary conditions these boundary conditions are not used, or, indeed, they do not play any role at all. Still, the numerical results agree between the exact and effective boundary conditions and this shows that at sufficiently low frequencies and not too high thickness-to-width ratio (but this ratio is as high as 0.25 in the numerical results) the boundary conditions on the sides are of no importance. Consequently, the use of the mixed boundary conditions in the exact solution is no 


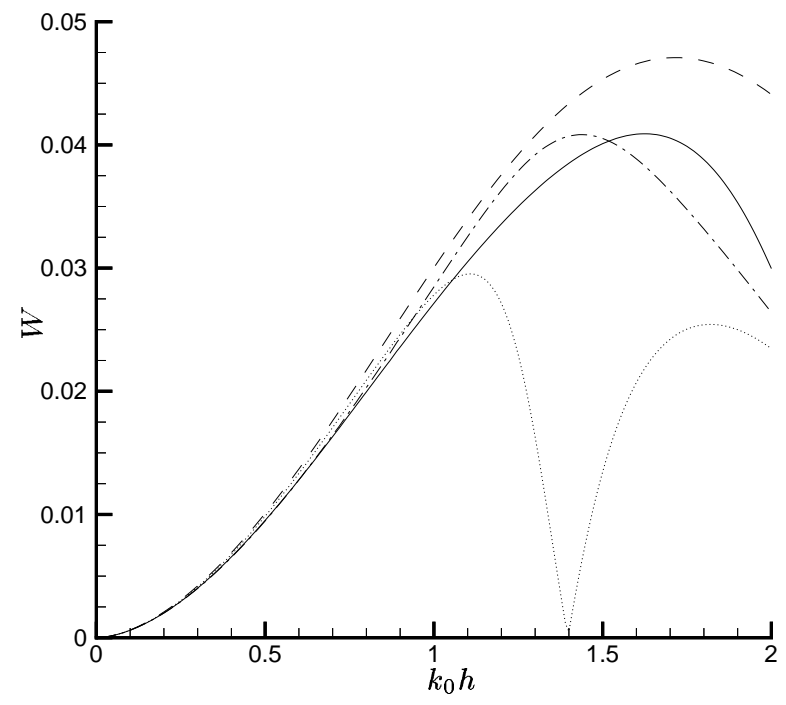

Fig. 4. The dimensionless displacement $W$ at $x=0, z=0$ as a function of frequency for $h / a=0.5$ and four cases: exact (fulldrawn), first order (dashed), second order (dash-dotted), and third order (dotted).

restriction of importance and very similar results are expected for other boundary conditions (with the restrictions on frequency and thickness-to-width).

\section{CONCLUDING REMARKS}

The $2 \mathrm{D}$ inplane problem with a piezoelectric strip actuator of finite width on top of an elastic halfspace is considered. The problem is solved both exactly, employing mixed boundary conditions on the sides of the strip to enabling Fourier series representations, and with effective boundary conditions, where no boundary conditions on the sides are needed. Three different orders of the effective boundary conditions are investigated. The lowest order is a simple traction boundary condition, but the higher order ones become progressively more complicated with also tangential derivatives involved.

The numerical results show that at sufficiently low frequencies all three effective boundary conditions give solutions that agree well with the exact solution. The higher order boundary conditions give good solutions for somewhat higher frequencies, but it is doubtful if the extra effort needed is worthwhile. In many practical applications the frequencies are low (in terms of the thickness of the piezoelectric strip), and then the lowest order effective boundary condition should be very useful. For instance, it should be straightforward to implement in a FEM progam.

\section{ACKNOWLEDGMENT}

The support of the Chinese Academy of Sciences and the National Natural Science Foundation of China $(10134020,9027003)$ that made possible the visit by the second author (B.Z.) to Chalmers University of Technology is gratefully acknowledged.

\section{REFERENCES}

[1] S.V. Gopinathan, V.V. Varadan, and V.K. Varadan. A review and critique of theories for piezoelectric laminates. Smart Mater. Struct., 9:24-48, 2000.

[2] R.C. Batra, X.Q. Liang, and J.S. Yang. The vibration of a simply supported rectangular elastic plate due to piezoelectric actuators. Int. J. Solids Structures, 33:1597-1618, 1996.

[3] H.F. Tiersten. Electroelastic equations for electroded thin plates subjected to large driving voltages. J. Appl. Phys., 74:33893393, 1993.

[4] J.-X. Gao, Y.-P. Shen, and J. Wang. Three dimensional analysis for free vibration of rectangular composite laminates with piezoelectric layers. J. Sound Vibr, 213:383-390, 1998.

[5] J. Wang and J. Yang. Higher-order theories of piezoelectric plates and applications. Appl. Mech. Rev., 53:87-99, 2000.

[6] H.S. Tzou. Piezoelectric Shells. Kluwer Academic Publishers, Dordrecht, The Netherlands, 1993.

[7] J. Kim, V.V. Varadan, and V.K. Varadan. Finite element modeling of structures including piezoelectric active devices. Int. J. Num. Methods Eng., 40:1-16, 1996.

[8] G. Johansson and A.J. Niklasson. Approximate dynamic boundary conditions for a thin piezoelectric layer. Int. J. Solids Struct., 40:3477-3492, 2003.

[9] B. Zhang, A. Boström, and A.J. Niklasson. Antiplane shear waves from a piezoelectric strip actuator: exact versus effective boundary condition solutions. Smart Mater. Struct., 13:161168, 2004.

[10] B.A. Auld. Acoustic Fields and Waves in Solids. Robert E. Krieger Publishing Company, Malabar, Florida, 1990.

[11] H.F. Tiersten. Linear piezoelectric plate vibrations. Plenum Press, New York, 1969. 